\title{
Successful desensitization of pemetrexed-induced anaphylaxis in a patient with malignant mesothelioma
}

Jeonghwan Youk ${ }^{1}$, Hyunkyung Park ${ }^{1}$, Kwang Nam Jin², Hyun Jong Moon ${ }^{3}$, Min-Suk Yang ${ }^{4}$, Ki Hwan Kim², and Jin-Soo Kim ${ }^{4}$

${ }^{1}$ Department of Internal Medicine, Seoul National University Hospital, Seoul; Departments of ${ }^{2}$ Radiology, ${ }^{3}$ Cardiothoracic Surgery, and ${ }^{4}$ Internal Medicine, Seoul Metropolitan Government Seoul National University Boramae Medical Center, Seoul, Korea

Received: July 31, 2015

Revised : September 24, 2015

Accepted: September 24, 2015

Correspondence to

Jin-Soo Kim, M.D.

Department of Internal Medicine, Seoul Metropolitan Government Seoul National University Boramae Medical Center, 20 Boramae-ro 5-gil, Dongjak-gu, Seoul 07061, Korea

Tel: $+82-2-870-3202$

Fax: $+82-2-870-2866$

E-mail: gistmd@gmail.com
To the Editor,

Pemetrexed is a multi-targeted anti-folate commonly used in patients with malignant pleural mesothelioma (MPM) and non-squamous non-small cell lung cancer. With the significant improvement of the median survival time in cisplatin plus pemetrexed (cis/ pem) arm (12.1 months vs. 9.3 months) in an EMPHACIS (Evaluation of Mesothelioma in a Phase III Trial of Pemetrexed with Cisplatin) trial [1], cis/pem has been the standard of care as a first line therapy for advanced MPM. As with other chemotherapeutic drugs, pemetrexed can induce a hypersensitivity reaction despite a relatively lower frequency $(<1 \%)$. To date, two case reports have been described related to the pemetrexed-induced anaphylaxis $[2,3]$. However, there was no report of desensitization and treatment continuation after the serious adverse reaction. Here, we described a stage IV mesothelioma patient who successfully overcame pemetrexed-induced anaphylaxis with systematic desensitization.

A 52-year-old female complained of feeling hardness on her left breast for half a year. A computed tomography (CT) scan of her chest showed a huge $(12.3 \times 4.5 \times 14.4 \mathrm{~cm})$ mass involving pas rietal pleura and left pectoralis major muscle (Fig. 1). With enlarged left supraclavicular lymph nodes, chest wall biop- sy led a diagnosis of stage IV mesothelioma. Standard cis/pem chemotherapy was recommended for treatment after the multidisciplinary discussion.

After six cycles of cis/pem chemotherapy, her tumor showed partial response to subsequent CT scans (Fig. 1). When she was admitted for the seventh cycle of cis/pem, she complained of sudden dizziness, chills and a subsequent generalized skin rash 5 minutes after being infused with pemetrexed. Her blood pressure was 86/49, while the oxygen saturation dropped to $89 \%$. With the suspicion of an anaphylactic reaction, pemetrexed infusion was immediately halted and $0.5 \mathrm{mg}$ epinephrine (1:1,000) was injected subcutaneously. After 20 minutes, she was free of symptoms and her blood pressure returned to normal (109/59).

Due to her remarkable response to cis/pem chemotherapy, which is the only known effective treatment for her deadly disease, we planned to continue the chemotherapy with a systematic desensitization protocol. The systematic desensitization protocol described in Table 1 was prepared, and three solutions were delivered in 12 consecutive steps. Despite a mild skin rash developing, we were able to give her a full dose of pemetrexed without eliciting another severe hypersensitivity reaction. At present, she has finished the 13th cycle 

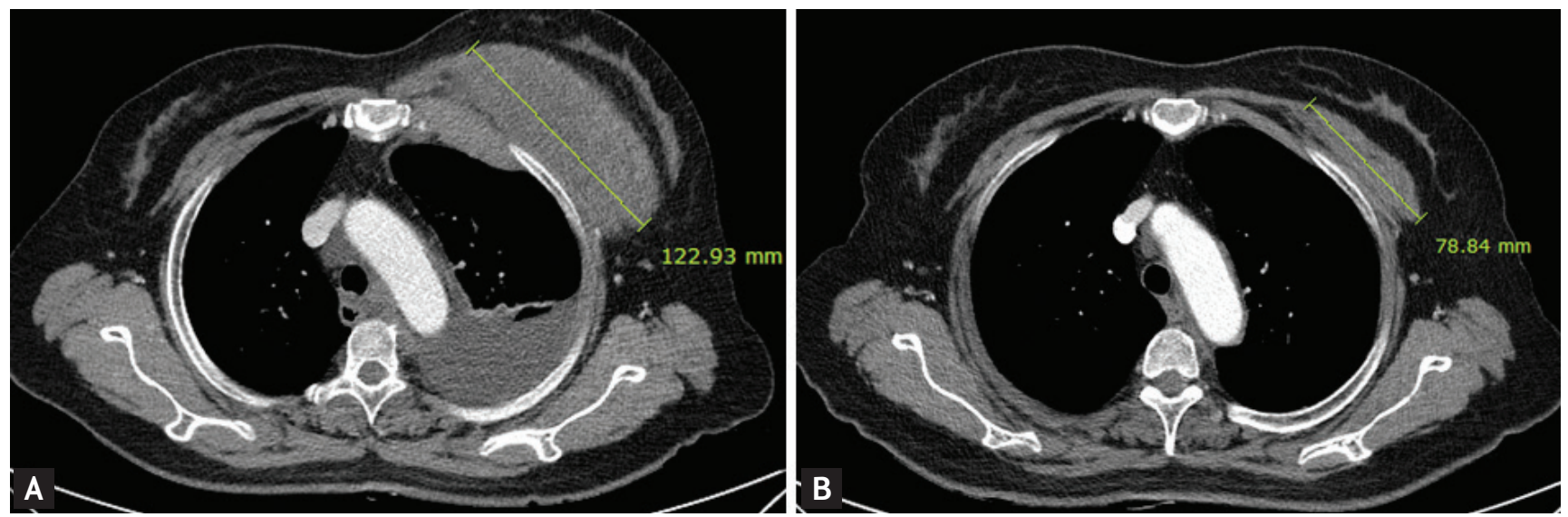

Figure 1. Chest computed tomography was performed (A) before the first cycle of chemotherapy, and (B) after the sixth cycle of chemotherapy. As shown, a good partial response was achieved after the sixth cycle of chemotherapy.

Table 1. Desensitization protocol for pemetrexed

\begin{tabular}{lccc}
\hline Order & Solution & $\begin{array}{c}\text { Infusion rate, } \\
\mathrm{mL} / \mathrm{hr}\end{array}$ & $\begin{array}{c}\text { Infusion } \\
\text { time, min }\end{array}$ \\
\hline 1 & $\mathrm{C}$ & 5 & 15 \\
2 & $\mathrm{C}$ & 10 & 15 \\
3 & $\mathrm{C}$ & 20 & 15 \\
4 & $\mathrm{C}$ & 50 & 15 \\
5 & $\mathrm{C}$ & 100 & 17.25 \\
6 & $\mathrm{~B}$ & 20 & 15 \\
\hline 7 & $\mathrm{~B}$ & 50 & 15 \\
8 & $\mathrm{~B}$ & 100 & 19.5 \\
9 & $\mathrm{~A}$ & 20 & 15 \\
10 & $\mathrm{~A}$ & 50 & 15 \\
11 & $\mathrm{~A}$ & 100 & 15 \\
12 & $\mathrm{~A}$ & 200 & 15.6 \\
\hline
\end{tabular}

Solution A: pemetrexed $815 \mathrm{mg}+$ normal saline $100 \mathrm{~mL}$; Solution B: pemetrexed $40.75 \mathrm{mg}+$ normal saline $50 \mathrm{~mL}$; Solution C: pemetrexed $4.075 \mathrm{mg}+$ normal saline $50 \mathrm{~mL}$.

of the cis/pem treatment with the desensitization protocol, while maintaining a good partial response.

This is the first case of pemetrexed anaphylaxis to be reported in Korea, and also the first report of the successful desensitization of pemetrexed hypersensitivity to date. Physicians often encounter situations where the early interruption of an effective chemotherapeutic drug should be considered, because of a severe hypersensitivity reaction. Anaphylaxis, as well as other hypersensitivity reactions, is caused by mediators, such as histamine, prostaglandin, and leukotriene, from mast cells and basophils. These mediators are released when allergen specific immunoglobulin E (IgE) attaches to mast cells or the mast cells are activated by direct extrinsic stimuli. Rapid desensitization is applicable to both IgE dependent anaphylaxis and IgE independent anaphylaxis. This strategy evokes temporary tolerance to a drug. In the case of IgE-dependent anaphylaxis, several studies have been performed to show the mechanism of the desensitization. Some plausible explanations are as follows [4]: the Fc receptor of IgE, expressed on mast cells, disappears after repeated exposure to the antigen, or there is molecular stabilization of the membrane bound IgE receptors without the disappearance of the receptors.

Researchers at Brigham and Women's Hospital (BWH) have reported a 12-step desensitization protocol [5]. In the study, 98 patients underwent a total of 413 desensitizations and only $6 \%$ of the patients experienced severe hypersensitivity reactions. Moreover, these patients had less severe reactions than their initial reactions, which were completely manageable. In our center, the modified BWH desensitization protocol has been commonly performed and was successfully applied in this case.

By this successful continuation of pemetrexed chemotherapy with rapid desensitization, physicians can prevent unwanted early interruptions of this important drug for cancer patients.

Keywords: Pemetrexed; Desensitization, immunologic; Anaphylaxis 


\section{Conflict of interest}

No potential conflict of interest relevant to this article was reported.

\section{REFERENCES}

1. Manegold C. Pemetrexed (Alimta, MTA, multitargeted antifolate, LY231514) for malignant pleural mesothelioma. Semin Oncol 2003;30(4 Suppl 10):32-36.

2. Capelle H, Birnbaum J, Tomasini P, et al. Anaphylactic reaction to pemetrexed: a case report. J Pharm Pharm
Sci 2014;17:229-230.

3. Shah BK, Hewett Y. Pemetrexed-induced anaphylaxis. Acta Oncol 2013;52:881.

4. Castells M, Sancho-Serra Mdel C, Simarro M. Hypersensitivity to antineoplastic agents: mechanisms and treatment with rapid desensitization. Cancer Immunol Immunother 2012;61:1575-1584.

5. Castells MC, Tennant NM, Sloane DE, et al. Hypersensitivity reactions to chemotherapy: outcomes and safety of rapid desensitization in 413 cases. J Allergy Clin Immunol 2008;122:574-580. 